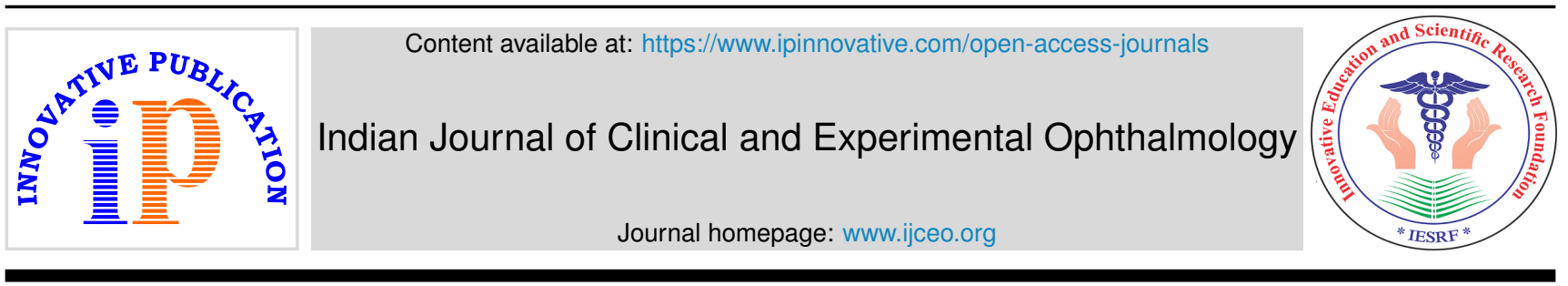

Original Research Article

\title{
Retro-pupillary iris fixated intraocular lens: A boon to aphakia
}

\author{
Ravi Bypareddy ${ }^{1}$, Kamakshi N Moger ${ }^{1}$, Vinutha Mohan Kulkarni' ${ }^{1}$, \\ Subramanya K Giliyar ${ }^{1, *}$, Deeksha Bekal ${ }^{1}$, B L Sujatha Rathod ${ }^{1}$ \\ ${ }^{1}$ Dept. of Ophthalmology, Minto Regional Institute of Ophthalmology, Bangalore Medical College and Research Institute, \\ Bengaluru, Karnataka, India
}

\section{A R T I C L E I N F O}

\section{Article history:}

Received 03-03-2021

Accepted 06-05-2021

Available online 30-06-2021

\section{Keywords:}

Aphakia

Anterior vitrectomy

Retro-pupillary iris fixated intraocular lens

\begin{abstract}
A B S T R A C T
Aim: To evaluate post operative visual outcomes and complications of retro-pupillary iris fixated Intracoular lens (IOL) in aphakia.

Materials and Methods: This is a prospective interventional case study conducted at our tertiary eye care centre from March 2018 to February 2019. All monocular aphakias secondary to any cause with no capsular support, good iris diaphragm support and BCVA of better than 6/60 were included. Patients who required combined procedures like trabeculectomy and retinal procedure were excluded. Patients with poor endothelial count/ corneal decompensation, any posterior segment pathologies, pre existing glaucoma and any form of uveitis were also excluded from the study. Pre operative visual acuity, slit lamp examination and fundus examination were carried out. Anterior vitrectomy and retropupillary fixation of iris claw lens were done. The primary outcome was to assess the post operative visual acuity and secondary outcome was to analyse post operative complications at various intervals up to 6 months after surgery.

Results: Our study comprised of thirty eyes of 30 aphakic patients. Retropupillary Iris fixated IOL was inserted as primary intraocular lens in 18 patients $(60 \%)$ and secondary intraocular lens in 12 patients $(40 \%)$. Baseline best corrected visual acuity was $0.831 \pm 0.66 \log$ MAR. Four patients had light perception at baseline. The mean best corrected visual acuity was $0.77 \pm 0.35 \log$ MAR at month $1,0.64 \pm 0.36 \log$ MAR at month 3 and $0.53 \pm 0.33 \log$ MAR at month 6 . Mean intraocular pressure at baseline was $16 \mathrm{mmHg}$. Mean intraocular pressure at month 1,3 and 6 were $17 \mathrm{mmHg}, 16 \mathrm{mmHg}$, and $16 \mathrm{mmHg}$ respectively. Among the complications, twelve eyes $(40 \%)$ had significant ovalisation of pupil at post operative month 1 which persisted at month 6, two eyes $(6.66 \%)$ had transient ocular hypertension (OHT), and none of the eyes progressed to glaucoma. Choroidal detachment was noted in 2 eyes (6.66\%), CME in 3 eyes $(9.99 \%)$, 1 eye $(3.33 \%)$ had retinal detachment and 2 eyes $(6.66 \%)$ had one haptic disenclavation. Among the complications, ovalisation of pupil was the most common observed one.

Conclusion: Retro-pupillary Iris fixated IOL is an effective, safe and simple procedure for management of aphakia in eyes with no posterior capsular support.

(C) This is an open access article distributed under the terms of the Creative Commons Attribution License (https://creativecommons.org/licenses/by/4.0/) which permits unrestricted use, distribution, and reproduction in any medium, provided the original author and source are credited.
\end{abstract}

\section{Introduction}

Cataract surgery is the most common cause of aphakia. During a complicated cataract surgery, zonular dialysis or posterior capsular tear/dehiscence can cause inadequate capsular support and difficulty to implant "in the bag" IOL or ciliary sulcus supported IOL. ${ }^{1}$ There are various

\footnotetext{
* Corresponding author.

E-mail address: subramanyakota@gmail.com (S. K. Giliyar).
}

intraocular lenses available to overcome this problem and to provide good vision to the patient. Angle supported anterior chamber intraocular lens (ACIOL), ${ }^{2}$ anterior chamber(AC) iris-claw lens, ${ }^{3}$ retropupillary iris fixated IOL, ${ }^{4,5}$ and Scleral-fixated IOL (SFIOL) ${ }^{6}$ are the various options available for surgical correction of aphakia. ACIOL insertion is technically easy, but it carries complications like pseudophakic bullous keratopathy, uveitis, damage to angle 
structures, glaucoma, ${ }^{7}$ cystoid macular edema, pupillary block, fibrosis of haptics and hence contraindicated in patients with glaucoma, shallow anterior chamber and corneal endothelial pathologies. Anterior chamber Iris claw IOL also carries complications like Corneal endothelial damage, ${ }^{8}$ secondary glaucoma and pupillary block. Hence came this idea of retropupillary iris fixated IOL, which is more simple, effective and safer technique than ACIOL or Anterior chamber Iris claw IOL.

SFIOLs are another mode to treat aphakia and it has the advantage of being placed near focal point of the eye. But the fixation procedure is time consuming with difficult intraocular manipulation. It is characterised by certain complications such as IOL tilt, optic capture of iris, vitreous hemorrhage, IOL decentration/dislocation, knot exposure, suture breakage and retinal detachment. ${ }^{9}$

Given the simplicity of the procedure, shorter learning curve, ${ }^{10}$ lesser complications and effectiveness, retropupillary Iris fixated IOL have become popular in the management of aphakia. In this technique, haptics of the poly methyl methacrylate (PMMA) IOL are anchored to posterior surface of the iris, placing them far from the corneal endothelium and irido-corneal angle, which theoretically reduces the risk for trabecular meshwork damage and endothelial damage when compared to ACIOL/Anterior chamber iris claw IOL. There is no general consensus about the best placement of iris claw lens, however study reported AC implantation causes higher endothelial cell loss (ECL) compared to retropupillary iris fixated IOL. ${ }^{11}$

The purpose of this non comparative study was to analyse the visual outcomes and complications after the retropupillary iris fixated IOL implantation for correction of aphakia, both as primary or secondary procedure.

\section{Materials and Methods}

This is a prospective interventional study conducted at our tertiary eye care centre. Thirty patients who underwent retropupilary iris fixated IOL from March 2018 to February 2019 were enrolled. Patients were fully informed about the risks and benefits of the surgery and informed consent was obtained. Study was conducted in accordance with tenets of Declaration of Helsinki. All monocular aphakias secondary to any cause with no capsular support, good iris diaphragm support and BCVA of better than 6/60 were included. Patients who required combined procedures like trabeculectomy and retinal procedure were excluded. Patients with poor endothelial count/ corneal decompensation, any posterior segment pathologies, pre existing glaucoma and any form of uveitis were also excluded from the study. Preoperative data were collected on patient demographics, history of prior ocular surgery, cause for aphakia, associated ocular diseases, uncorrected distance visual acuity(UCVA), best corrected distance visual acuity(BCVA), refraction, intraocular pressure, slit lamp examination, dilated fundus evaluation and biometry were recorded. Post operative data were obtained on UCVA, BCVA, clinical signs of ECL, IOL position, IOP, pigment dispersion, gonioscopy(wherever necessary) and complications.

\subsection{Surgical technique}

All patients underwent standard procedure of choice - Anterior vitrectomy and Iris claw lens implantation. Standard SRK/T formula was used to calculate the IOL power. Under peribulbar anesthesia, all the surgeries were performed by the single experienced surgeon, using the same surgical protocol(SK). In case of primary IOL surgery, same surgical wound was used for insertion of IOL. In cases of secondary IOL implantation, the pre-existing sclerocorneal tunnel was opened. Three limbal side ports at 3, 4 and 9 o'clock were made, AC maintainer was secured and anterior vitrectomy was done to ensure that there were no residual vitreous strands in the AC or wound. Remnants of anterior and posterior capsule along with the cortex and irido-vitreal adhesions were removed. Peripheral iridectomy (PI) was performed using the vitrector between 11 and 2 o' clock. Intracameral $0.1 \mathrm{ml}$ of pilocarpine nitrate $0.5 \%$ (Aurocarpine, Madurai, India) was used to constrict the pupil. Viscoelastic (Hydroxy propyl methyl cellulose 2\%) was injected into the AC. Using iris-claw holding forceps, iris-claw IOL was introduced in the AC, and the haptics were rotated to 3 and 9 o' clock, using a Sinskey hook. The optic was held in the center of the pupil with forceps, and the haptic was dipped under the iris surface. The bump of the haptic should be visible on the iris surface, and using a Sinskey hook through the side port, the mid peripheral iris was gently tapped at the center of the haptic to enclave the IOL to the posterior iris surface. Once the haptic was enclaved, a dimple was visible on the iris surface, and the other haptic was enclaved in a similar way. After enclavation, IOL was tapped to check for the IOL stability. Viscoelastic was removed and side ports were hydrated. Scleral incision was sutured with interrupted 100 nylon and conjunctiva was sutured with $8-0$ polyglactin (Vicryl). Postoperatively, all the patients were prescribed topical prednisolone acetate $1 \%$ and moxifloxacin $0.5 \%$ four times/day, tapered over a period of 6 weeks. Patients were asked to come for follow-up at 1 day, 1 week, 1 month, 3 months, 6 months, 1 year, and 6 monthly thereafter.

\subsection{Statistical analysis}

Statisitcal analysis was performed using the SPSS version 16 (SPSS/IBM Inc., Chicago, Illinois, USA). Snellen CDVA was converted to $\log$ MAR units for the analysis. Visual acuity of hand movement and Counting finger close to face was assigned the equivalent of 3 and $2 \log$ MAR units, 
respectively. ${ }^{12,13}$ Descriptive analysis was carried out, and the data were analyzed using paired t-test. A value of $\mathrm{P}<$ 0.05 was considered statistically significant.

\section{Results}

The study comprised of thirty eyes of 30 patients with monocular aphakia. The mean age was $54 \pm 6$ years (range 20-60 years). The IOLs were inserted during primary lens procedure for 18 patients $(60 \%)$ and as secondary lens procedure in 12 patients $(40 \%)$. There was no gender predilection. The parameters assessed during follow up were visual acuity and various post-operative complications.

Intraoperative PC rent with no PC support following cataract surgery was the most common etiology of aphakia. (Table 1) Baseline best corrected visual acuity was $0.831 \pm 0.66 \log$ MAR. The mean best corrected visual acuity was $0.77 \pm 0.35 \log$ MAR at one month $0.64 \pm 0.36 \log$ MAR at three months and $0.53 \pm 0.33 \log$ MAR at six months respectively. (Table 2) Improvement in the visual acuity was statistically significant compared to preoperative visual acuity ( $\mathrm{P}$ value $<0.05)$.

Mean intraocular pressure at baseline was $16 \mathrm{mmHg}$. Mean intraocular pressure at month 1, 3 and 6 were $17 \mathrm{mmHg}, \quad 16 \mathrm{mmHg}$, and $16 \mathrm{mmHg}$. Among the complications, twelve eyes $(40 \%)$ had significant ovalisation of pupil at post operative month 1 which persisted at month 6 , two eyes $(6.66 \%)$ had transient ocular hypertension (OHT), and none of the eyes progressed to glaucoma. Choroidal detachment was noted in 2 eyes $(6.66 \%), \mathrm{CME}$ in 3 eyes $(9.99 \%), 1$ eye $(3.33 \%)$ had retinal detachment and 2 eyes $(6.66 \%)$ had one haptic disenclavation. There were no complications intraoperatively and all the IOLs were well centered at the end of the procedure.

Pupillary distortion (oval shaped pupil) was the most common complication noted in 12 patients(40\%). It was followed by pigment clumping over the IOL (27\%). Elevated IOP and macular edema were noted in 3 patients $(10 \%)$. Rise in IOP was managed adequately with topical anti-glaucoma medication. At each visit IOP was measured and it was observed that IOP had decreased adequately. Iris chaffing at the enclavation site of the IOL was noted in 2 patients $(6.66 \%)$. All complications are depicted in Table 3.

Table 1: Etiology of aphakia

\begin{tabular}{lcc} 
Causes & Number & Percentage \\
Traumatic Cataract & 3 & 10 \\
Intraoperative pc rent with & 22 & 73 \\
no PC support & & \\
$\begin{array}{l}\text { Pex with NDP } \\
\text { Mature cataract with } \\
\text { subluxation }\end{array}$ & 2 & 7 \\
\hline
\end{tabular}

Table 2: Visual acuity changes after surgery

\begin{tabular}{lccc} 
BCVA & $\mathbf{6 / 6 - 6 / 1 8}$ & $\mathbf{6 / 2 4 - ~} \mathbf{6 / 6 0}$ & $<\mathbf{6 / 6 0}$ \\
Preoperative & 14 & 9 & 7 \\
At 1 month & 10 & 14 & 6 \\
At 3 month & 13 & 12 & 5 \\
At 6 month & 20 & 5 & 5 \\
\hline
\end{tabular}

Table 3: Intraoperative and post operative complications

\begin{tabular}{lcc} 
Complications & Numbers & Percentage \\
Pupil distortion & 12 & 40 \\
Iris chaffing at enclavation & 2 & 7 \\
site & 8 & 27 \\
Pigment clump over iol & 3 & 10 \\
Secondary glaucoma & 3 & 10 \\
Macular edema & 1 & 3 \\
Retinal detachment & 1 & 3 \\
Vitreous hemorrhage & & \\
\hline
\end{tabular}

\section{Discussion}

Aphakia is one of the complications of cataract surgery due to inadequate posterior capsular support. Surgical correction of aphakia is associated with better binocular visual acuity than spectacle correction or contact lens use. Various intraocular lenses are available to correct aphakia like anterior chamber IOLs (ACIOLs), AC iris claw IOL, scleral fixated IOLs and retropupillary iris fixated IOL. ${ }^{4,5}$ ACIOLs have more incidence of endothelial decompensation ${ }^{8}$ whereas SFIOLs need more experience to master. Basically in our study we evaluated post operative visual outcome and complications in aphakic patients after retropupillary iris claw placement. Each of the available method has its own pros and cons. The retropupillary placement of iris claw lens has advantages of true posterior chamber IOL with lesser intra and post operative complications and best possible visual rehabilitation. The visual outcome was best attained in the group of post cataract surgery patients compared to traumatic cataract. It has another advantage of lesser risk of endothelial decompensation.

In our study, patients were in the age group of 20-60 years with the mean age of 54 \pm-6 years which is comparable with the study done by Mansoori et al. ${ }^{1}$ where the mean age was 54.64 years.

In our study, at the end of 6 months follow up, 25 patients had BCVA of $>6 / 18,5$ patients had BCVA of $>6 / 60$ and other 5 patients with $>6 / 60$ due to macular edema, retinal detachment and vitreous haemorrhage. Patients with macular edema slowly recovered vision after treatment.

The most common complication noted in our study was pupil distortion (ovalisation of pupil) in 12 patients $(40 \%)$. In a study done by Mansoori et al. ${ }^{1}$ noticed 20 cases of irregular pupil(20\%). Similarly in a study done by Tomy $\mathrm{R}$ et al noticed mild temporal ovalisation in $14.2 \%$ and Gonnerman et al. ${ }^{13}$ in their study reported pupil irregularity 
in $13.9 \%$.

In our study, elevated intraocular pressure of $22 \mathrm{mmHg}$ was noted in 3 patients $(10 \%)$ on $1^{\text {st }}$ postoperative day which was controlled with antiglaucoma medications and permanent rise was not noted which is comparable with the study done by Toro et al. ${ }^{14}$ In our study we also noted pigment clumping over the IOL in 8 patients $(27 \%)$.

In our study, cystoid macular edema was observed in 3 patients $(10 \%)$ which attributed to low vision. According to the study done by Toro et al. ${ }^{14}$ who observed macular edema 4-12 weeks after cataract surgery and contributed to decreased vision which later improved on treatment. Study conducted by Tomy $\mathrm{R}$ et al. ${ }^{14}$ found macular edema in 3 patients which accounted for $8.57 \%$.

In our study, we noted 1 case of retinal detachment and 1 case of vitreous haemorrhage which also contributed to low vision at 6 months follow up.

The limitations of our study was that endothelial cell counts were not done preoperatively owing to the study done by Gicquel et al. ${ }^{11}$ in which they compared $\mathrm{AC}$ iris claw IOL and retropupillary iris claw IOL and observed lower endothelial cell loss in posterior chamber implantation significantly. Another limitation was lesser sample size of 30 patients.

\section{Conclusion}

The retropupillary iris claw IOL implantation provided good visual outcome with favourable complication rate. It has the advantage of posterior chamber lens position near to nodal point of eye, surgery is easy to master and faster technique with no significant corneal damage. It is the safe and effective method to correct aphakia in patients with no posterior capsule support.

\section{Source of Funding}

None.

\section{Conflict of Interest}

The authors declare that there is no conflict of interest.

\section{References}

1. Mansoori T, Agraharam SG, Sannapuri S, Manwani S, Balakrishna N. Surgical outcomes of retropupillary-fixated iris-claw intraocular lens. $\quad J$ Curr Ophthalmol. 2020;32(2):149-53. doi:10.4103/joco.joco_92_20.

2. Lieb WA, Guerry D. 3rd Anterior chamber lenses; for refractive correction of unilateral aphakia. Am J Ophthalmol. 1957;44:579-98.

3. Lett KS, Chaudhuri PR. Visual outcomes following Artisan aphakia iris claw lens implantation. Eye. 2011;25(1):73-6. doi:10.1038/eye.2010.146.

4. Kelkar A, Shah R, Vasavda V, Kelkar J, Kelkar S. Primary iris claw IOL retrofixation with intravitreal triamcinolone acetonide in cases of inadequate capsular support. Int Ophthalmol. 2017;38:111-8. 101:10.1007/s:0792-017-(2467-9

5. Jare NM, Kesari AG, Gadkari SS, Deshpande MD. The posterior irisclaw lens outcome study: 6-month follow-up. Indian J Ophthalmol. 2016;64:878-83.

6. Chan TC, Lam JK, Jhanji V, Li EY. Comparison of outcomes of primary anterior chamber versus secondary scleral-fixated intraocular lens implantation in complicated cataract surgeries. Am J Ophthalmol. 2015;159:221-6.

7. Sawada T, Kimura W, Kimura T, Suga H, Ohte A, Yamanishi S. Long-term follow-up of primary anterior chamber intraocular lens implantation. J Cataract Refractive Surg. 1998;24(11):1515-20. doi:10.1016/s0886-3350(98)80176-4.

8. Peralba RT, Lamas-Francis D, Sarandeses-Diez T, Martínez-Pérez L, Rodríguez-Ares T. Iris-claw intraocular lens for aphakia: Can location influence the final outcomes? J Cataract Refract Surg. 2018;44(7):818-26. do1:10.1016/].jcrs.2018.05.010.

9. Kwong YY, Yuen HK, Lam RF, Lee VY, Rao SK, Lam DS. Comparison of outcomes of primary scleral-fixated versus primary anterior chamber intraocular lens implantation in complicated cataract surgeries. Ophthalmology. 2007;114:80-5.

10. Madhivanan N, Sengupta S, Sindal M, Nivean PD, Kumar MA, Ariga M. Comparative analysis of retropupillary iris claw versus scleralfixated intraocular lens in the management of post-cataract aphakia. Indian J Ophthalmol. 2019;67(1):59-63. do1:10.4103/1jo.1]0_326_18

11. Gicquel JJ, Guigou S, Bejjani RA, Briat B, Ellies P, Dighiero P. Ultrasound biomicroscopy study of the Verisyse aphakic intraocular lens combined with penetrating keratoplasty in pseudophakic bullous keratopathy. J Cataract Refract Surg. 2007;33(3):455-64. do1:10.1016/1.jcrs.2006.11.017.

12. Appel SD, Brilliant RL. The low vision examination. In: Brilliant RL, editor. Essentials of Low Vision Practice. Boston: ButterworthHeinemann; 1999. p. 20-46.

13. Gonnermann J, Klamann MKJ, Maier AK, Rjasanow J, Joussen AM, Bertelmann E, et al. Visual outcome and complications after posterior iris-claw aphakic intraocular lens implantation. J Cataract Refract Surg. 2012;38(12):2139-43. doi:10.1016/1.jcrs.2012.07.035.

14. Toro MD, Longo A, Avitabile T, Nowomiejska K, Gagliano C, Tripodi $\mathrm{S}$, et al. Five-year follow-up of secondary iris-claw intraocular lens implantation for the treatment of aphakia: Anterior chamber versus retropupillary implantation. PLOS ONE. 2019;14(4):e0214140. doi:10.1371/journal.pone.0214140

\section{Author biography}

Ravi Bypareddy, Assistant Professor

Kamakshi N Moger, Senior Resident

Vinutha Mohan Kulkarni, Post Graduate

Subramanya K Giliyar, Assistant Professor

Deeksha Bekal, Post Graduate

B L Sujatha Rathod, Director

Cite this article: Bypareddy R, Moger KN, Kulkarni VM, Giliyar SK, Bekal D, Rathod BLS. Retro-pupillary iris fixated intraocular lens: A boon to aphakia. Indian J Clin Exp Ophthalmol 2021;7(2):415-418. 